\title{
The Static Approximation to B Meson Mixing using Light Domain-Wall Fermions: Perturbative Renormalization and Ground State Degeneracies
}

\author{
Norman H. Christ, Thomas T. Dumitrescu*, Oleg Loktik \\ Department of Physics, Columbia University, New York, NY 10027, USA \\ E-mail: nhc@phys.columbia.edu, td2135@columbia.edu, \\ oleg@phys.columbia.edu
}

\section{Taku Izubuchi}

RIKEN-BNL Research Center, Brookhaven National Laboratory, Upton, NY 11973, USA and Institute for Theoretical Physics, Kanazawa University, Kanazawa, Ishikawa 920-1192, Japan

E-mail: izubuchi@hep.s.kanazawa-u.ac.jp

\section{RBC-UKQCD Collaboration}

We discuss the theoretical input into the current RBC-UKQCD calculation of $f_{B_{d, s}}$ and $B_{B_{d, s}}$ using a smeared static heavy quark propagator, light domain-wall quarks and the Iwasaki gauge action. We present the complete one-loop, mean-field improved matching of heavy-light current and fourfermion lattice operators onto the static continuum theory renormalized in $\overline{\mathrm{MS}}$ (NDR). The large degeneracies present in a static calculation are addressed, and a method for extracting $f_{B}$ and $B_{B}$ using only box sources is described; implications for future calculations are discussed.

The XXV International Symposium on Lattice Field Theory

July 30-4 August 2007

Regensburg, Germany

\footnotetext{
* Speaker.
} 


\section{Introduction}

Precision measurements of the CKM matrix put the Standard Model to a stringent test and constrain possible physics beyond it. Using the measured frequency of $B_{q}-\bar{B}_{q}, q \in\{d, s\}$ oscillations to determine the CKM matrix elements $\left|V_{t q}\right|$ requires a reliable lattice calculation of the non-perturbative $B_{q}-\bar{B}_{q}$ mixing matrix elements $\frac{8}{3} m_{B_{q}}^{2} f_{B_{q}}^{2} B_{B_{q}}$. A $2+1$ flavor, unquenched calculation of $f_{B_{q}}$ and $B_{B_{q}}$ has been carried out by the RBC-UKQCD collaboration in the infinite heavy quark mass limit using light domain-wall fermions on a $(2 \mathrm{fm})^{3}$ spatial volume [1,2]; this is currently being extended to a $(3 \mathrm{fm})^{3}$ spatial volume and towards physical light quark masses [3]. In the following, we discuss the perturbative lattice-continuum matching of the operators relevant for the RBC-UKQCD calculation, following in part the detailed discussion in Refs. [2, 4]. We also point out the subtle degeneracy of heavy-light meson ground states, and discuss its implications for the extraction of $f_{B}$ and $B_{B}$ from lattice correlation functions.

\section{Action and Feynman Rules}

The heavy $b$ quark is described by an improved lattice version of the static limit of heavy quark effective theory with smeared, $\mathrm{SU}(3)$-projected gauge links $\bar{V}_{0}(\vec{x}, t)$ to reduce noise:

$$
S_{\text {static }}=\sum_{\vec{x}, t} \bar{h}(\vec{x}, t+a)\left[h(\vec{x}, t+a)-\bar{V}_{0}^{\dagger}(\vec{x}, t) h(\vec{x}, t)\right] .
$$

The SU(3) projection (discussed in Ref. [2]) simplifies perturbative calculations by allowing the smeared gauge links to be expanded in terms of an effective gauge field $B_{0}^{a}(\vec{x}, t)$; in momentum space $B_{0}^{a}(q)=h_{\mu}(q) A_{\mu}^{a}(q)$, where $A_{\mu}^{a}(q)$ is the physical gauge field and $h_{\mu}(q)$ is a form factor depending on the smearing scheme. We focus on one of the two schemes used in the RBC-UKQCD calculation (one-level APE blocking with parameter $\alpha=1$ ), resulting in a heavy quark gluon vertex

$$
Y_{\mu}^{a}\left(k, k^{\prime}\right)=-i g_{0} T^{a} \delta_{\mu 0} e^{-i\left(k_{0}+k_{0}^{\prime}\right) / 2} \rightarrow \bar{Y}_{\mu}^{a}\left(k, k^{\prime}\right)=-i g_{0} T^{a} h_{\mu}(q) e^{-i\left(k_{0}+k_{0}^{\prime}\right) / 2},
$$

where $g_{0}$ is the bare lattice coupling, $q$ is the gluon momentum, and $h_{\mu}(q)$ is given by

$$
h_{\mu}(q)=\left(h_{0}(q), h_{j}(q)\right)=\left(1-\frac{2}{3} \sum_{l=1}^{3} \sin ^{2}\left(\frac{q_{l}}{2}\right), \frac{2}{3} \sin \left(\frac{q_{0}}{2}\right) \sin \left(\frac{q_{j}}{2}\right)\right) .
$$

The heavy quark two-gluon vertex and the heavy quark propagator are given in Ref. [4].

The light quarks are described by the domain-wall fermion action. Each light flavor is represented by a $(4+1)$-dimensional Wilson-style fermion field $\psi_{s}(\vec{x}, t)$ where $1 \leq s \leq N$ labels the coordinate in the fifth dimension. The physical quark field $q(\vec{x}, t)$ is constructed from chiral surface states at $s=1$ and $s=N$ via $q(\vec{x}, t)=P_{R} \psi_{1}(\vec{x}, t)+P_{L} \psi_{N}(\vec{x}, t)$. The domain-wall height $M_{5}$ is a fixed parameter of the theory; we set $M_{5}=1.8$ to match the RBC-UKQCD calculation. A detailed description of domain-wall fermions and their perturbative treatment for our choice of gauge action is given in Ref. [4] and references therein, especially Ref. [5]. In the perturbative calculation the light quark masses were set to zero and the size $N$ of the fifth dimension was taken to be large, resulting in an exact chiral symmetry as $N \rightarrow \infty$. The gluons were described by the Iwasaki gauge action, whose Feynman rules are given in Ref. [4]. 


\section{Perturbative Lattice-Continuum Matching at One-Loop}

The full QCD operators relevant for the extraction of $f_{B}$ and $B_{B}$, defined in $\overline{\mathrm{MS}}(\mathrm{NDR})$ at the scale $\mu_{b}=m_{b}$ of the $b$ quark mass, are the axial vector current $A_{\rho}=\bar{b} \gamma_{\rho} \gamma_{5} q$ and the parity-even part of the $\Delta B=2$ vector-axial four-quark operator:

$$
\left[\bar{b} \gamma^{\rho}\left(1-\gamma_{5}\right) q\right]\left[\bar{b} \gamma_{\rho}\left(1-\gamma_{5}\right) q\right] \rightarrow O_{V V+A A}=\left(\bar{b} \gamma^{\rho} q\right)\left(\bar{b} \gamma_{\rho} q\right)+\left(\bar{b} \gamma^{\rho} \gamma_{5} q\right)\left(\bar{b} \gamma_{\rho} \gamma_{5} q\right)
$$

We match these operators at the scale $\mu_{b}$ to lattice operators in the static effective theory (described in Sec. 2) at the lattice scale $a^{-1}$ via the continuum version of the static effective theory renormalized at a scale $\mu$. Throughout our one-loop calculation we choose to set $\mu=a^{-1}$; in the RBC-UKQCD calculation, the lattice scale is given by $a^{-1}=1.62 \mathrm{GeV}$. The full QCD operators are related to continuum static operators by

$$
\begin{gathered}
A_{\rho}\left(\mu_{b}\right)=C_{A}\left(\mu_{b}, \mu\right) \widetilde{A}_{\rho}(\mu)+\mathscr{O}\left(\Lambda_{\mathrm{QCD}} / \mu_{b}\right) \\
O_{V V+A A}\left(\mu_{b}\right)=Z_{1}\left(\mu_{b}, \mu\right) \widetilde{O}_{V V+A A}(\mu)+Z_{2}\left(\mu_{b}, \mu\right) \widetilde{O}_{S S+P P}(\mu)+\mathscr{O}\left(\Lambda_{\mathrm{QCD}} / \mu_{b}\right) .
\end{gathered}
$$

In terms of the static quark and antiquark fields $h^{( \pm)}(x)=e^{ \pm i m_{b} v \cdot x}(1 \pm \psi) b(x) / 2$ and for $m_{b} \rightarrow \infty$,

$$
\begin{gathered}
\widetilde{A}_{\rho}=\bar{h}^{(+)} \gamma_{\rho} \gamma_{5} q, \\
\widetilde{O}_{V V+A A}=2\left(\bar{h}^{(+)} \gamma^{\rho} q\right)\left(\bar{h}^{(-)} \gamma_{\rho} q\right)+2\left(\bar{h}^{(+)} \gamma^{\rho} \gamma_{5} q\right)\left(\bar{h}^{(-)} \gamma_{\rho} \gamma_{5} q\right), \\
\widetilde{O}_{S S+P P}=2\left(\bar{h}^{(+)} q\right)\left(\bar{h}^{(-)} q\right)+2\left(\bar{h}^{(+)} \gamma_{5} q\right)\left(\bar{h}^{(-)} \gamma_{5} q\right) .
\end{gathered}
$$

The static effective action discussed in Sec. 2 describes $h^{(+)}$with $v=(1, \overrightarrow{0})$, corresponding to a stationary meson. The constants $C_{A}\left(\mu_{b}, \mu\right)$ and $Z_{1,2}\left(\mu_{b}, \mu\right)$ are known at one-loop; they are summarized in Ref. [2]. Using the latest PDG values for $\alpha_{s}^{\overline{\mathrm{MS}}}\left(m_{Z}\right)$ and $m_{b}$, and running the coupling down at four-loops with the physical number of flavors to determine $\alpha_{s}^{\overline{\mathrm{MS}}}\left(\mu_{b}\right)$ and $\alpha_{s}^{\overline{\mathrm{MS}}}(\mu)$ we obtain $C_{A}=1.057, Z_{1}=0.934, Z_{2}=-0.151$.

We now describe the matching $\widetilde{A}_{\rho}(\mu)=\widetilde{C}_{A}\left(\mu, a^{-1}\right) a^{-3} A_{\rho}^{\text {lat }}$ of the heavy-light axial currents $\widetilde{A}_{\rho}(\mu)$ and $A_{\rho}^{\text {lat }}$ (which is dimensionless) in the continuum and lattice versions of the static effective theory. Results for the four-fermion operators are summarized at the end of this section. We compare the correlation function $\langle(\bar{h}(x) \Gamma q(x)) h(y) \bar{q}(z)\rangle$ in both theories; in this discussion only one heavy quark field $h^{(+)} \equiv h$ enters. For the axial current $\Gamma=\gamma_{\rho} \gamma_{5}$, but the light quark chiral symmetry and the heavy quark spin symmetry $h \rightarrow e^{-i \phi_{j} \varepsilon_{j k l} \sigma_{k l}} h$ of both the continuum and the lattice theory render the matching $\Gamma$-independent. At one-loop and for small external quark momenta $p \simeq 0$ the continuum and lattice correlation functions are

$$
\langle(\bar{h} \Gamma q) h \bar{q}\rangle=\frac{Z_{h}}{i p_{0}} \Gamma(1+\delta V) \frac{Z_{2}}{i \not p}, \quad\langle(\bar{h} \Gamma q) h \bar{q}\rangle_{\mathrm{lat}}=\frac{Z_{h}^{\mathrm{lat}}}{i p_{0}} \Gamma\left(1+\delta V^{\mathrm{lat}}\right) \frac{\left(1-w_{0}^{2}\right) Z_{w} Z_{2}^{\text {lat }}}{i \not p},
$$

where the Feynman diagrams contributing at one-loop are shown in Fig. 1. All Z-factors have values $1+\mathscr{O}\left(\alpha_{s}\right)$, and the vertex corrections $\delta V, \delta V^{\text {lat }}$ are $\mathscr{O}\left(\alpha_{s}\right)$ and $\Gamma$-independent as noted above. 
The continuum quantities are known [4]; we focus on the lattice correlation function: $w_{0}=1-M_{5}$ is a domain-wall fermion specific constant, and an overlap factor $1-w_{0}^{2}$ connecting the five-dimensional and physical quark fields is present even at tree level. The light quark wavefunction renormalization $Z_{w} Z_{2}^{\text {lat }}$ due to Fig. 1 (a) and (b) was calculated in Ref. [5]. $Z_{2}^{\text {lat }}$ can be viewed as the four-dimensional wavefunction renormalization, while $Z_{w}$ renormalizes the overlap factor $1-w_{0}^{2}$. Due to tadpoles, the one-loop correction to $Z_{w}$ is enormous. As described in Ref. [5], this is remedied by reorganizing the perturbation series according to the

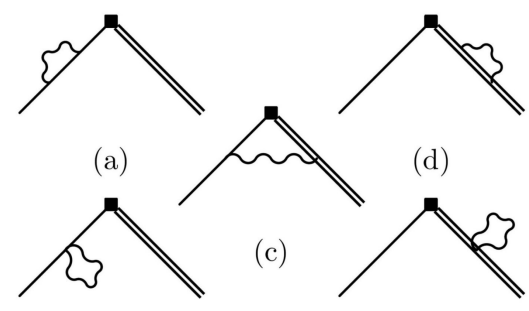

(b)

(e)

Figure 1: One-Loop Corrections to the Heavy-Light Axial Current mean-field approach, resulting in the prescriptions $M_{5} \rightarrow \widetilde{M}_{5}=M_{5}-4(1-u), w_{0} \rightarrow w_{0}^{\mathrm{MF}}=1-\widetilde{M}_{5}$ and $q^{\text {lat }} \rightarrow q^{\text {lat, } \mathrm{MF}}=u^{-1 / 2} q^{\text {lat }}$ to be made throughout the calculation; here $u=P^{1 / 4}$ where $P$ is the measured average plaquette (for the RBC-UKQCD calculation $u=0.8757$ ) and the superscript 'MF' identifies mean-field improved quantities. We calculate the matching factor $\widetilde{C}_{A}\left(\mu, a^{-1}\right)$ using both the usual continuum $\overline{\mathrm{MS}}$ coupling and a mean-field

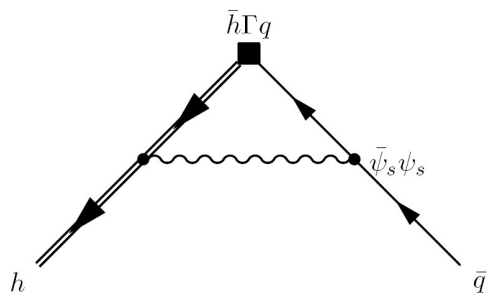

Figure 2: One-Loop Vertex Correction to the Heavy-Light Axial Current improved version, enabling an estimate of $\mathscr{O}\left(\alpha_{s}^{2}\right)$ corrections. $\alpha_{s}^{\overline{\mathrm{MS}}}(\mu)$ was obtained by running down to the $c$ quark mass with the physical number of flavors and back up to $\mu$ using only three dynamical flavors to match the RBC-UKQCD $2+1$ flavor calculation: $\alpha_{s}^{\overline{\mathrm{MS}}}(\mu)=0.326$ and $\alpha_{s}^{\mathrm{MF}}(\mu)=0.177$. The calculation of the vertex correction $\delta V^{\text {lat }}$ in Fig. 1 (c) and the heavy quark wavefunction renormalization $Z_{h}^{\text {lat }}$ in Fig. 1 (d) and (e) is straightforward $[2,4]$. Infrared divergences only occur in QEDlike diagrams and are regulated by a gluon mass $\lambda$ which cancels from the matching factor. Furthermore, only the unsmeared $\delta_{\mu 0}$ part of $h_{\mu}(q)$ in Eq. (2.3) gives rise to infrared divergences; the sine functions in the smeared part of $h_{\mu}(q)$ cancel all infrared divergent loop propagators. A generic feature of domain-wall fermion perturbation theory is the appearance of correlation functions $\left\langle q(-p) \bar{\psi}_{s}(p)\right\rangle$, $\left\langle\psi_{s}(-p) \bar{q}(p)\right\rangle$ connecting external four-dimensional quarks to five-dimensional quarks propagating in loops, as shown in Fig. 2. A subtlety pointed out in Ref. [6] is that the correct renormalization prescription for $Z_{h}^{\text {lat, } M F}$ includes the linearly divergent heavy quark mass renormalization:

$$
Z_{h}^{\text {lat, } \mathrm{MF}}=1-\left.i \frac{\partial \Sigma\left(p_{0}\right)}{\partial p_{0}}\right|_{p_{0}=0}+\Sigma\left(p_{0}=0\right)
$$

where the heavy quark self energy $\Sigma\left(p_{0}\right)$ itself is not affected by mean-field improvement. Comparing the correlation functions in Eq. (3.7) after mean-field improvement gives a matching factor

$$
\widetilde{C}_{A}\left(\mu, a^{-1}\right)=\frac{\sqrt{u}}{\sqrt{\left(1-\left(w_{0}^{\mathrm{MF}}\right)^{2}\right) Z_{w}^{\mathrm{MF}}}} Z_{A}^{\mathrm{MF}}\left(\mu, a^{-1}\right), \quad Z_{A}^{\mathrm{MF}}\left(\mu, a^{-1}\right)=1+\frac{\alpha_{s}}{3 \pi}(-1.584)
$$


The overall factor $Z_{\Phi}\left(\mu_{b}, a^{-1}\right)=C_{A}\left(\mu_{b}, \mu\right) \widetilde{C}_{A}\left(\mu, a^{-1}\right)$ relating the axial currents in full QCD and the lattice static effective theory, computed using both $\alpha_{s}^{\overline{\mathrm{MS}}}(\mu)$ and $\alpha_{s}^{\mathrm{MF}}(\mu)$, is $Z_{\Phi}^{\overline{\mathrm{MS}}}\left(\mu_{b}, a^{-1}\right)=$ $0.902, Z_{\Phi}^{\mathrm{MF}}\left(\mu_{b}, a^{-1}\right)=0.961$. While the one-loop result is small and reliable, the large difference between $\alpha_{s}^{\overline{\mathrm{MS}}}(\mu)$ and $\alpha_{s}^{\mathrm{MF}}(\mu)$ induces a $\sim 7 \%$ systematic error ultimately warranting nonperturbative renormalization.

For completeness, we quote the lattice-continuum matching constants (calculated in Refs. [2, 4]) for the four-fermion operators in Eqs. (3.5) and (3.6). For $i \in\{V V+A A, S S+P P\}$ and at one-loop

$$
\widetilde{O}_{i}(\mu)=\frac{u}{\left(1-\left(w_{0}^{\mathrm{MF}}\right)^{2}\right) Z_{w}^{\mathrm{MF}}} Z_{i}^{\mathrm{MF}}\left(\mu, a^{-1}\right) a^{-6} O_{i}^{\mathrm{lat}}, \quad Z_{V V+A A}^{\mathrm{MF}}=1+\frac{\alpha_{s}}{4 \pi}(-4.462), \quad Z_{S S+P P}^{\mathrm{MF}}=1
$$

where the $O_{i}^{\text {lat }}$ are dimensionless. Since the coefficient $Z_{2}$ of $\widetilde{O}_{S S+P P}$ in Eq. (3.3) is $\mathscr{O}\left(\alpha_{s}\right)$, only the domain-wall overlap factors contribute to the lattice-continuum matching for this operator. While formally inconsistent, we use the one-loop mean-field improved values of the overlap factors throughout to ensure tadpole-safety. Combining Eqs. (3.3) and (3.10) we get:

$$
\begin{gathered}
O_{V V+A A}=Z_{V A}\left(\mu_{b}, a^{-1}\right) a^{-6} O_{V V+A A}^{\text {lat }}+Z_{S P}\left(\mu_{b}, a^{-1}\right) a^{-6} O_{S S+P P}^{\text {lat }}, \\
Z_{V A}^{\overline{\mathrm{MS}}}=0.902, \quad Z_{V A}^{\mathrm{MF}}=0.769, \quad Z_{S P}^{\overline{\mathrm{MS}}}=-0.123, \quad Z_{S P}^{\mathrm{MF}}=-0.133 .
\end{gathered}
$$

\section{Ground State Degeneracies of Static-Light Mesons and $f_{B}, B_{B}$ on the Lattice}

Let $H$ be the Hamiltonian corresponding to the full lattice action in Sec. 2. For any $t$, the heavy quark action in Eq. (2.1) is invariant under $h(\vec{x}) \rightarrow e^{i \theta(\vec{x})} h(\vec{x})$ for a set of $V / a^{3}$ parameters $\theta(\vec{x})$, where $V=L^{3}$ is the spatial lattice volume. If $\Theta(\vec{x})$ is the generator corresponding to $\theta(\vec{x})$ then

$$
[\Theta(\vec{x}), h(\vec{y})]=h(\vec{x}) \delta_{\vec{x} \vec{y}}, \quad[\Theta(\vec{x}), \bar{h}(\vec{y})]=-\bar{h}(\vec{x}) \delta_{\vec{x} \vec{y}}, \quad[\Theta(\vec{x}), \Theta(\vec{y})]=0, \quad[\Theta(\vec{x}), H]=0 .
$$

Simultaneously diagonalize $H$ and all $\Theta(\vec{x})$. Since Eq. (4.1) implies that $h(\vec{x})$ and $\vec{h}(\vec{x})$ raise and lower the eigenvalues of $\Theta(\vec{x})$ by 1 , and the charge conjugation invariance of QCD implies $\Theta(\vec{x})|0\rangle=0$, the spectrum of $\Theta(\vec{x})$ contains $\mathbb{Z}$. Define the unit-norm state $|B(\vec{x})\rangle$ to be the lowest energy state with the quantum numbers of a $B$ meson which also satisfies $\Theta(\vec{y})|B(\vec{x})\rangle=\delta_{\vec{x} \vec{y}}|B(\vec{x})\rangle$. Thus $\langle B(\vec{x}) \mid B(\vec{y})\rangle=\delta_{\vec{x} y}$, and we can interpret these states as having the heavy quark localized at a fixed lattice site with the light quark smeared out around it. Since $T(\hat{i}) \Theta(\vec{x}) T(\hat{i})^{-1}=\Theta(\vec{x}+\hat{i})$, where $T(\hat{i})$ is a lattice translation by $a$ in the spatial direction $\hat{i}$, all $B$ meson ground states $|B(\vec{x})\rangle$ are degenerate. We also define total spatial momentum eigenstates $\left|\widetilde{B}\left(\vec{k}_{l}\right)\right\rangle$, where $l_{i} \in \mathbb{Z}(i=1,2,3)$ :

$$
\left|\widetilde{B}\left(\vec{k}_{l}\right)\right\rangle=\sqrt{2 a^{3}} \sum_{\vec{x}} e^{-i \vec{k}_{l} \cdot \vec{x}}|B(\vec{x})\rangle, \quad \vec{k}_{l}=\frac{2 \pi}{L}\left(l_{1}, l_{2}, l_{3}\right),-\frac{L}{2 a}<l_{i} \leq \frac{L}{2 a}, \quad\left\langle\widetilde{B}\left(\vec{k}_{l^{\prime}}\right) \mid \widetilde{B}\left(\vec{k}_{l}\right)\right\rangle=2 V \delta_{l^{\prime} l}
$$

As $a \rightarrow 0, V \rightarrow \infty$, these states reduce to continuum momentum eigenstates $|\widetilde{B}(\vec{p})\rangle^{\mathrm{c}}$ with conventional static effective theory normalization ${ }^{\mathrm{c}}\left\langle\widetilde{B}\left(\vec{p}^{\prime}\right) \mid \widetilde{B}(\vec{p})\right\rangle^{\mathrm{c}}=2(2 \pi)^{3} \delta^{(3)}\left(\vec{p}^{\prime}-\vec{p}\right)$. In the $m_{b} \rightarrow \infty$ limit, these states only differ from the corresponding full QCD states by a factor of $\sqrt{m_{B}}$. Thus: 


$$
\begin{aligned}
f_{B} \sqrt{m_{B}} \equiv\left\langle 0\left|A_{0}(\overrightarrow{0}, 0)\right| \widetilde{B}(\vec{p}=\overrightarrow{0})\right\rangle^{\mathrm{c}} & =Z_{\Phi}^{\mathrm{MF}} a^{-3}\langle 0| A_{0}^{\text {lat }}(\overrightarrow{0}, 0)\left(\sqrt{2 a^{3}} \sum_{\vec{x}}|B(\vec{x})\rangle\right)= \\
& =\sqrt{2} Z_{\Phi}^{\mathrm{MF}} a^{-3 / 2}\left\langle 0\left|A_{0}^{\text {lat }}(\overrightarrow{0}, 0)\right| B(\overrightarrow{0})\right\rangle \equiv \sqrt{2} Z_{\Phi}^{\mathrm{MF}} a^{-3 / 2} \Phi_{B}^{\text {lat }} .
\end{aligned}
$$

In complete analogy to the above, we can construct $\bar{B}$ meson ground states states $|\bar{B}(\vec{x})\rangle$. Using these and Eq. (3.11), the calculation of the $B-\bar{B}$ mixing matrix element $\frac{8}{3} m_{B}^{2} f_{B}^{2} B_{B}=\left\langle\bar{B}\left|O_{V V+A A}\right| B\right\rangle$ is reduced to the calculation of the lattice quantities $\left\langle\bar{B}(\overrightarrow{0})\left|O_{i}^{\text {lat }}(\overrightarrow{0}, 0)\right| B(\overrightarrow{0})\right\rangle, i \in\{V V+A A, S S+P P\}$.

The degeneracy of the states $|B(\vec{x})\rangle$ complicates the extraction of $\Phi_{B}^{\text {lat }}$ and $\left\langle\vec{B}(\overrightarrow{0})\left|O_{i}^{\text {lat }}(\overrightarrow{0})\right| B(\overrightarrow{0})\right\rangle$, since even a large time separation of source and sink may not project onto a unique $B$ meson ground state: different combinations of the $|B(\vec{x})\rangle$ may enter the correlation functions used for calculating the matrix elements and those used for normalization. To see this, consider the extraction of $\Phi_{B}^{\text {lat }}$; we now work exclusively in the lattice theory. Define local and smeared $B$ meson interpolation operators $A_{0}^{L}(\vec{x}, t)=\bar{h}(\vec{x}, t) \gamma_{0} \gamma_{5} q(\vec{x}, t), A_{0}^{S}(t)=\sum_{\vec{y} \in \Delta V} \sum_{\vec{z} \in \Delta V} \bar{h}(\vec{y}, t) \gamma_{0} \gamma_{5} q(\vec{z}, t)$, where $\Delta V$ is a fixed subvolume of $V$ and the smeared operators are Coulomb gauge fixed. From experience, locallocal correlation functions in the static effective theory are prohibitively noisy; instead calculate the local-smeared and smeared-smeared correlation functions. Inserting a complete set of states $\sum_{\vec{w}}|B(\vec{w})\rangle\langle B(\vec{w})|+$ (higher energy states) with the correct quantum numbers, we have as $t \rightarrow \infty$ :

$$
\begin{gathered}
\mathscr{C}^{L S}(t) \equiv \sum_{\vec{x} \in V}\left\langle 0\left|A_{0}^{L}(\vec{x}, t) A_{0}^{S}(0)^{\dagger}\right| 0\right\rangle=\Phi_{B}^{\mathrm{lat}} e^{-m_{B}^{*} t}\left(\sum_{\vec{w} \in V}\left\langle B(\vec{w})\left|\sum_{\vec{y} \in \Delta V} \sum_{\vec{z} \in \Delta V} \bar{q}(\vec{y}, 0) \gamma_{0} \gamma_{5} h(\vec{z}, 0)\right| 0\right\rangle\right), \\
\mathscr{C}^{S S}(t) \equiv\left\langle 0\left|A_{0}^{S}(t) A_{0}^{S}(0)^{\dagger}\right| 0\right\rangle=e^{-m_{B}^{*} t}\left(\sum_{\vec{w} \in V}\left|\left\langle B(\vec{w})\left|\sum_{\vec{y} \in \Delta V} \sum_{\vec{z} \in \Delta V} \bar{q}(\vec{y}, 0) \gamma_{0} \gamma_{5} h(\vec{z}, 0)\right| 0\right\rangle\right|^{2}\right)
\end{gathered}
$$

where $m_{B}^{*}$ is the unphysical mass of the lattice $B$ meson. Since $\mathscr{C}^{S S}(t)$ contains a sum over squares, the use of a naive ratio $\sim \mathscr{C}^{L S}(t) / \sqrt{\mathscr{C}^{S S}(t)}$ requires a translationally invariant wall source $\Delta V=V$ to project onto the unique state of zero-momentum. In this case the sums over $\vec{w}$ only give a factor of $V / a^{3}$ and $\Phi_{B}^{\text {lat }}=\mathscr{C}^{L S}(t) / \sqrt{\mathscr{C}^{S S}(t) e^{-m_{B}^{*} t} V / a^{3}}$. To remedy the poor overlap of the wall source with the $B$ meson ground state - especially on large lattices - consider a fixed box source and a series of box sinks summed over an entire timeslice to project onto zero momentum; this approach also allows more general types of smearing, such as the use of an atomic wavefunction. Let $\widetilde{A}_{0}^{S}(\vec{w}, t)=\sum_{\vec{y} \in \Delta V_{\vec{w}}} \sum_{\vec{z} \in \Delta V_{\vec{w}}} \bar{h}(\vec{y}, t) \gamma_{0} \gamma_{5} q(\vec{z}, t)$ where $\Delta V_{\vec{w}}$ is a box of fixed size located at $\vec{w}$ and $\Delta V_{\overrightarrow{0}}=\Delta V, \widetilde{A}_{0}^{S}(\overrightarrow{0}, t)=A_{0}^{S}(t)$. Define a corresponding smeared-smeared correlation function and insert a complete set of momentum eigenstates $\frac{1}{2 V} \sum_{\vec{k}_{l}}\left|\widetilde{B}\left(\vec{k}_{l}\right)\right\rangle\left\langle\widetilde{B}^{(} \vec{k}_{l}\right) \mid+$ (higher energy states); then as $t \rightarrow \infty$,

$\mathscr{C}^{\widetilde{S S}}(t) \equiv \sum_{\vec{w}}\left\langle 0\left|\widetilde{A_{0}^{S}}(\vec{w}, t) A_{0}^{S}(0)^{\dagger}\right| 0\right\rangle=\frac{e^{-m_{B}^{*} t}}{2 V} \sum_{\vec{w}}\left\langle 0\left|\widetilde{A_{0}^{S}}(\vec{w}, t)\right| \widetilde{B}(\overrightarrow{0})\right\rangle\left\langle\widetilde{B}(\overrightarrow{0})\left|A_{0}^{S}(0)^{\dagger}\right| 0\right\rangle=\frac{e^{-m_{B}^{*} t}}{2 a^{3}}\left|\left\langle\widetilde{B}(\overrightarrow{0})\left|A_{0}^{S}(0)^{\dagger}\right| 0\right\rangle\right|^{2}$ 
Since $|\widetilde{B}(\overrightarrow{0})\rangle=\left(2 a^{3}\right)^{1 / 2} \sum_{\vec{w}}|B(\vec{w})\rangle$, we can rewrite the right side of Eq. (4.4) and obtain another ratio for $\Phi_{B}^{\text {lat }}$ which reaches a plateau more quickly due to the improved ground state overlap:

$$
\mathscr{C}^{L S}(t) e^{m_{B}^{*} t / 2} / \sqrt{\mathscr{C}^{\widetilde{S}}(t)}=\Phi_{B}^{\mathrm{lat}}\left\langle\widetilde{B}(\overrightarrow{0})\left|A_{0}^{S}(0)^{\dagger}\right| 0\right\rangle / \sqrt{\left|\left\langle\widetilde{B}(\overrightarrow{0})\left|A_{0}^{S}(0)^{\dagger}\right| 0\right\rangle\right|^{2}}=\Phi_{B}^{\mathrm{lat}} .
$$

The calculation of $\left\langle\bar{B}(\overrightarrow{0})\left|O_{i}^{\text {lat }}(\overrightarrow{0})\right| B(\overrightarrow{0})\right\rangle, i \in\{V V+A A, S S+P P\}$ is considerably simpler. Define

$$
\mathscr{C}_{O_{i}}(T, t) \equiv \sum_{\vec{x} \in V}\left\langle 0\left|\bar{A}_{0}^{S}(T) O_{i}^{\text {lat }}(\vec{x}, t) A_{0}^{S}(0)^{\dagger}\right| 0\right\rangle,
$$

where $\bar{A}_{0}^{S}(T)=\sum_{\vec{y} \in \Delta V} \sum_{\vec{z} \in \Delta V} \bar{q}(\vec{y}, T) \gamma_{0} \gamma_{5} h(\vec{z}, T)$. Proceeding as above, we have as $t, T-t \rightarrow \infty$ :

$$
\left\langle\bar{B}(\overrightarrow{0})\left|O_{i}^{\mathrm{lat}}(\overrightarrow{0}, 0)\right| B(\overrightarrow{0})\right\rangle=\mathscr{C}_{O_{i}}(T, t) / \mathscr{C}^{S S}(T)=\mathscr{C}_{O_{i}}(T, t) e^{m_{B}^{*} T / 2} / \sqrt{\mathscr{C}^{S S}(T-t) \mathscr{C} S S(t)} .
$$

Here no zero momentum projection is necessary; the use of $\mathscr{C}^{S S}$ for smaller time separations simply reduces noise. Using Eqs. (4.7) and (4.9) we can thus calculate $f_{B}$ and $B_{B}$ using only box sources and sinks. These are preferable to wall sources, whose poor ground state overlap led to late plateaus in the $V=(2 \mathrm{fm})^{3} \mathrm{RBC}-\mathrm{UKQCD}$ calculation and presents an even bigger problem for the ongoing extension to $V=(3 \mathrm{fm})^{3}$. It is worth emphasizing that this simple method relies on the particular properties of the static effective theory, and further such improvements might be possible.

\section{Acknowledgments}

We thank our RBC-UKQCD collaborators C. Albertus, Y. Aoki, P. A. Boyle, L. Del Debbio, J. M. Flynn, C. T. Sachrajda, A. Soni, and J. Wennekers. We gratefully acknowledge the support of BNL, Columbia University, the University of Edinburgh, PPARC, RIKEN, and the U.S. DOE.

\section{References}

[1] C. Albertus, Y. Aoki, P. A. Boyle, N. H. Christ, L. Del Debbio, T. T. Dumitrescu, J. M. Flynn, T. Izubuchi, O. Loktik, C. T. Sachrajda, A. Soni and J. Wennekers, $B-\bar{B}$ mixing with domain wall fermions, in these proceedings, POS (LATTICE 2007) 376.

[2] C. Albertus, Y. Aoki, P. A. Boyle, N. H. Christ, L. Del Debbio, T. T. Dumitrescu, J. M. Flynn, T. Izubuchi, O. Loktik, C. T. Sachrajda, A. Soni and J. Wennekers, Unquenched B meson decay constants and $B^{0}-\bar{B}^{0}$ mixing parameters from chiral latttice $Q C D$, in preparation.

[3] Y. Aoki for the RBC and UKQCD collaborations, Heavy-light matrix elements in static limit with domain wall fermions, in these proceedings, POS (LATTICE 2007) 345.

[4] O. Loktik and T. Izubuchi, Perturbative renormalization for static and domain-wall bilinears and four-fermion operators with improved gauge actions, Phys. Rev. D75 (2007) 034504 [hep-lat/0612022].

[5] S. Aoki, T. Izubuchi, Y. Kuramashi and Y. Taniguchi, Perturbative renormalization factors in domain-wall QCD with improved gauge actions, Phys. Rev. D67 (2003) 094502 [hep-lat/0206013].

[6] P. Boucaud, J. P. Leroy, J. Micheli, O. Pene and G. C. Rossi, A rigorous treatment of the lattice renormalization problem of $f_{B}$, Phys. Rev. D47 (1993) 1206-1218 [hep-lat/92080 04 ]. 